\title{
Identification, Analysis, and Confirmation of Seed Storability-Related Loci in Dongxiang Wild Rice (Oryza rufipogon Griff.)
}

\author{
Minmin Zhao ${ }^{1,+}$, Biaolin $\mathrm{Hu}^{2,+}{ }^{\circ}$, Yuanwei Fan ${ }^{3,+}+\ddagger$, Gumu Ding ${ }^{1}$, Wanling Yang ${ }^{4}$, Yong Chen ${ }^{4}$, Yanhong Chen ${ }^{1}$, \\ Jiankun Xie ${ }^{4, *}$ and Fantao Zhang ${ }^{1, *}$ \\ 1 College of Life Sciences, Jiangxi Normal University, Nanchang 330022, China; zhaomm0222@163.com (M.Z.); \\ dgm2207733038@163.com (G.D.); xiaofeiyan0508@163.com (Y.C.) \\ 2 Rice National Engineering Laboratory, Rice Research Institute, Jiangxi Academy of Agricultural Sciences, \\ Nanchang 330022, China; hubiaolin992@126.com \\ 3 College of Biological Sciences and Biotechnology, Beijing Forestry University, Beijing 100083, China; \\ yuanweifan@email.wustl.edu \\ 4 Jiangxi Provincial Key Lab of Protection and Utilization of Subtropical Plant Resources, \\ Nanchang 330022, China; yangwl3058@163.com (W.Y.); jxcydm@163.com (Y.C.) \\ * Correspondence: xiejiankun11@163.com (J.X.); zhang84004@163.com (F.Z.) \\ + Contributed equally to this work. \\ $\ddagger$ Current address: Department of Biology and Center for Engineering Mechanobiology, \\ Washington University in St. Louis, St. Louis, MO 63130, USA.
}

Citation: Zhao, M.; Hu, B.; Fan, Y.; Ding, G.; Yang, W.; Chen, Y.; Chen, Y.; Xie, J.; Zhang, F. Identification,

Analysis, and Confirmation of Seed Storability-Related Loci in Dongxiang Wild Rice (Oryza rufipogon Griff.). Genes 2021, 12, 1831. https://doi.org/ 10.3390 /genes12111831

Academic Editor: Bing Yang

Received: 31 October 2021

Accepted: 18 November 2021

Published: 19 November 2021

Publisher's Note: MDPI stays neutral with regard to jurisdictional claims in published maps and institutional affiliations.

Copyright: (C) 2021 by the authors. Licensee MDPI, Basel, Switzerland. This article is an open access article distributed under the terms and conditions of the Creative Commons Attribution (CC BY) license (https:// creativecommons.org/licenses/by/ $4.0 /)$.
Abstract: Dongxiang wild rice (Oryza rufipogon Griff.) (DXWR) has strong seed storability and identifying its elite gene resources may facilitate genetic improvements in rice seed storability. In this study, we developed two backcross inbred lines (BILs) populations, with DXWR as a common donor parent and two rice varieties (F6 and R974) as recipient parents. Bulked segregant analysis via whole genome sequencing (BSA-seq) was used to identify seed storability-related loci in the DXWR and F6 population. Two main genomic regions containing 18,550,000-20,870,000 bp on chromosome 4 and 7,860,000-9,780,000 bp on chromosome 9 were identified as candidate loci of DXWR seed storability; these overlapped partially with seed storability-related quantitative trait loci (QTLs) discovered in previous studies, suggesting that these loci may provide important regions for isolating the responsible genes. In total, 448 annotated genes were predicted within the identified regions, of which 274 and 82 had nonsynonymous and frameshift mutations, respectively. We detected extensive metabolic activities and cellular processes during seed storability and confirmed the effects of the seed storability-related candidate loci using four BILs from DXWR and R974. These results may facilitate the cloning of DXWR seed storability-related genes, thereby elucidating rice seed storability and its improvement potential.

Keywords: wild rice; seed storability; BSA-seq; genetic resource; rice improvement

\section{Introduction}

Rice (Oryza sativa L.) plays a vital role in food production and security globally [1]. However, rice seeds can lose their viability and vigour during storage very easily, which has caused serious yield losses. Seed aging and the decrease of vigour during storage have been the main problems of rice production [2,3]. In recent years, the problem of seed storage has become increasingly prominent in China owing to the continuous harvest of rice, especially in the humid and hot south area $[4,5]$. Currently, seed aging is delayed mainly by improving the storage conditions, which is costly and energy-consuming [6]. Breeding rice cultivars having strong seed storability is one of the most cost-effective and efficient ways to alleviate this problem. 
Seed storability is a complex quantitative trait and is affected by both external and internal factors [2,7]. Nowadays, external factors, such as chemical agents and the manipulation of temperature and humidity, are mainly used to prolong storage time; however, these processes are costly and rice seeds may be contaminated easily [6]. Therefore, it is of great significance to study how internal factors can be used to improve the seed storability of rice. The internal factors include the genetic background, fatty acid content, starch characteristics, antioxidants, etc. A number of physiological and biochemical studies have revealed that many changes can affect seed vigour, including membrane system, DNA, and protein damage; decreased protein synthesis ability; and the accumulation of excessive reactive oxygen species [8,9]. Meanwhile, the loss of three kinds of lipoxygenases (Lox1, Lox2, and Lox3) could delay the aging and deterioration of rice seeds, resulting in improved tolerance to storage $[6,10]$.

During the past decades, more than 70 quantitative trait loci (QTLs) related with seed storability have been identified in different rice accessions [11]. However, the traditional QTL analysis relies on the development of mapping populations, which is time-consuming and easily affected by genetic background noise [12]. Meanwhile, most of the QTL analyses on seed storability have been performed on populations derived from modern rice varieties, and most of the detected QTLs were isolated in large intervals, making subsequent finescale mapping difficult. Furthermore, the genetic resources of seed storability in modern rice varieties are limited, and some valuable genes of rice seed were lost during artificial selection and the long-term evolution process of rice [13]. Therefore, utilizing genetic resources from other rice species, especially in wild rice relatives, is significant for rice breeding strategies for seed storability improvement.

The advancement of next generation sequencing (NGS) technology provides an opportunity for exploring the genetic diversity among various rice accessions and its utilization in breeding programs. For example, Liang et al. applied NGS technology to identify a novel pi21 haplotype conferring basal resistance to rice blast disease [14]. Kitony et al. used NGS technology for population development and days to heading (DTH) QTLs mapping [15]. Reyes et al. utilized NGS technology for marker-assisted backcross breeding to introgress and stack the Gn1a and WFP alleles to improve rice cultivars [16]. On the other hand, bulked segregant analysis via whole genome sequencing (BSA-seq) is a fast and powerful tool to accurately identify QTLs associated with complex traits [17]. BSA-seq has been widely used in various plants, such as rice [18], cucumbers [19], and cotton [20]. However, there is limited research on the identification of elite QTLs from wild rice by BSA-seq. The Dongxiang wild rice, found in Dongxiang county, Jiangxi province, China, is the northernmost $\left(28^{\circ} 14^{\prime} \mathrm{N}\right)$ wild rice species globally. DXWR possesses many elite genetic resources related with tolerance against various abiotic stresses, such as cold, drought, salt, and hot temperature [21]. Additionally, DXWR has strong seed storability and harbours many valuable genetic resources [22]. However, the molecular mechanisms of the seed storability of DXWR remain poorly understood. Therefore, the main aim of the present study was to identify and analyse seed storability-related loci in DXWR using BSA-seq, which will be helpful in the fine-scale mapping and cloning of novel genetic resources improving the seed storability of rice.

\section{Materials and Methods}

\subsection{Plant Materials}

The parents DXWR and F6, and the derived backcross inbred lines (BILs) populations $\left(\mathrm{BC}_{2} \mathrm{~F}_{7} ; 252\right.$ lines) were used in the BSA-seq. Another BIL population $\left(\mathrm{BC}_{4} \mathrm{~F}_{6} ; 84\right.$ lines $)$ was derived from DXWR and R974. Using molecular marker background analysis, four BILs (BIL27, BIL49, BIL64, and BIL203) from the DXWR/R974 population were selected to analyse the effects of the candidate loci. In the two populations, the receptor parents, F6 and R974, are modern rice varieties and the common donor parent DXWR is an accession of Oryza rufipogon. The two populations were cultivated in the paddy fields of Jiangxi Normal 
University, Nanchang city, China in the summer of 2017. The seeds of the populations and parents were harvested, dried, and stored in a refrigerator at $-20^{\circ} \mathrm{C}$.

\subsection{Seed Storability Evaluation and Extreme Bulk Sample Construction}

For seed storability evaluation, the seeds were initially subjected to a 20-day artificial aging treatment in the dark at $42{ }^{\circ} \mathrm{C}$, in an environment with $88 \%$ humidity [11]. The treated seeds were then placed in a petri dish with a filter paper, were kept moist using sterile water, and then incubated in a constant temperature incubator at $32{ }^{\circ} \mathrm{C}$. The germination standard was based on the length of the germinated radicle or embryo reaching half the length of the seed [23]. After 5 days, the germination rates were counted. The germination rates $(\%)=$ number of germinated seeds $/$ total number of seeds $\times 100 \%$. For BSA-seq, 20 lines with high germination rates and 20 lines with low germination rates were selected to construct bulked-tolerant (TB-bulk) and bulked-intolerant (IB-bulk) BIL samples, respectively [24]. The experiment was repeated three times to ensure the correct phenotype. Statistical analysis was performed using Student's $t$-test.

\subsection{Sequence Libraries Construction and BSA-Seq}

Sequence libraries construction and BSA-seq were performed by Biomarker Technologies Corporation (Beijing, China). The procedure was carried out according to the protocol provided by Illumina (San Diego, CA, USA). Briefly, the qualified DNA was randomly fragmented into $350 \mathrm{bp}$ length by ultrasonic processor. Then, the DNA fragments were end repaired, and a A-tail was added to the $3^{\prime}$ end, followed by adapter ligation and purified. Subsequently, the sequencing libraries were constructed by PCR amplification and sequenced by Illumina Novaseq 6000 platform. The raw image data obtained by sequencing were transformed by base calling software (Illunima Casava v.1.8.2, San Diego, CA, USA) and stored in FASTQ format. Then, the raw reads were filtered to obtain clean reads for further bioinformatics analysis. The clean reads from four libraries were separately mapped to the Nipponbare MSU v7 reference genome (http:/ / rice.uga.edu/, accessed on June 13, 2021) using BWA software [25]. BSA-seq was conducted as previously described [26]. All SNPs and InDels variations were identified by Unified Genotype function of the Genome Analysis Toolkit (GATK) software [27], and the SnpEff software [28] was used to annotate the SNPs and InDels. The Picard Tool Mark Duplicates was used to remove the PCR duplicates (http:/ / sourceforge.net/projects / picard/, accessed on 15 June 2021). The detection of SNP/InDel was performed by the GATK software toolkit [27]. SNPs and Indels were filtered based on the GATK best practice guidelines [27]. Before performing association analysis, we filtered again in order to obtain high quality SNP/InDel sites [29]. SNP-index and InDel-index of each SNP and InDel position were calculated to identify the candidate loci associated with seed storability. To minimize false-positive sites, we used the positions of markers in the genome to fit the $\triangle$ SNP-index and $\Delta$ InDel-index values marked on the same chromosome using the DISTANCE method [30]. Additionally, a Euclidean distance (ED) measurement algorithm was also applied as previously described [31].

BLAST software [32] was used to annotate the genes in the identified candidate loci for seed storage tolerance with NT, NR [33], Swiss-Prot [34], GO [35], KEGG [36] and COG [37] databases. All annotated genes containing nonsynonymous and frameshift mutations in the identified candidate loci associated with seed storage tolerance were selected for further analysis.

\subsection{Molecular Marker Background Analysis}

Total genomic DNA was extracted from fresh leaves by the Plant Genomic DNA Extraction Kit (Sangon Biotech, Shanghai, China). PCR system and amplification procedure were performed as previously described [38]. PCR products were separated on 3\% agarose gels. SSR marker sequences were found in the Gramene website (www.gramene.org, accessed on 15 July 2021), and InDel marker sequences were designed by Primer3 Input (version 0.4.0) (https://bioinfo.ut.ee/primer3-0.4.0/, accessed on 16 July 2021). All of 
the molecular markers were synthesized by Sangon Biotech Company Limited (Shanghai, China) (Table S1).

\section{Results}

\subsection{Identification of BILs with Extreme Phenotypes for Seed Storability}

After the artificial aging treatment, the germination rate of DXWR was approximately $42.50 \%$, while that of $\mathrm{F} 6$ was $18.33 \%$. The germination rate differences between DXWR and F6 were significant (Figure 1a,b). Meanwhile, for each BIL of the population derived from DXWR and F6, the germination rates had obvious differences, ranging from $0 \%$ to $57.50 \%$, with an average value of $19.20 \%$ (Figure 1c). The distribution of the germination rates of the BILs had a skewed distribution. There were 118 BILs (46.83\%) had higher germination rates than that of the receptor parent $\mathrm{F} 6$, suggesting that an extensive introgression of genes from DXWR to F6 occurred in the population. Then, 20 BILs with high germination rates were grouped as TB-bulk sample and 20 BILs with low germination rates were grouped as IB-bulk sample for further BSA-seq analysis.

a

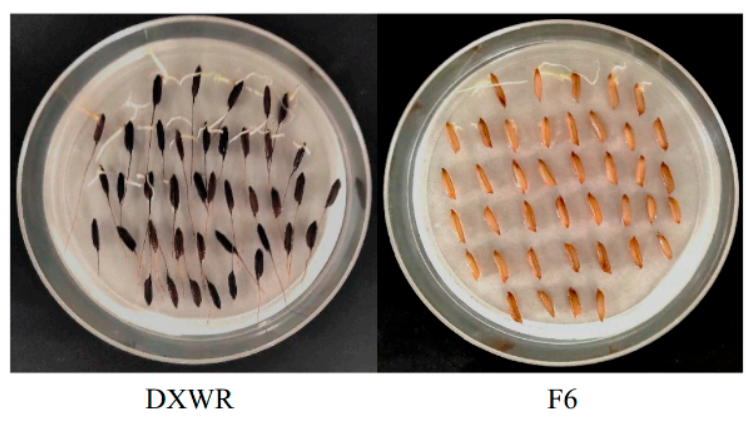

$\mathrm{b}$

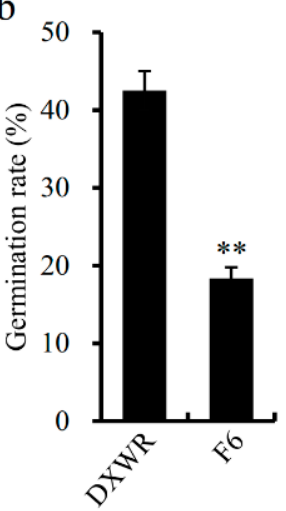

$\mathrm{c}$

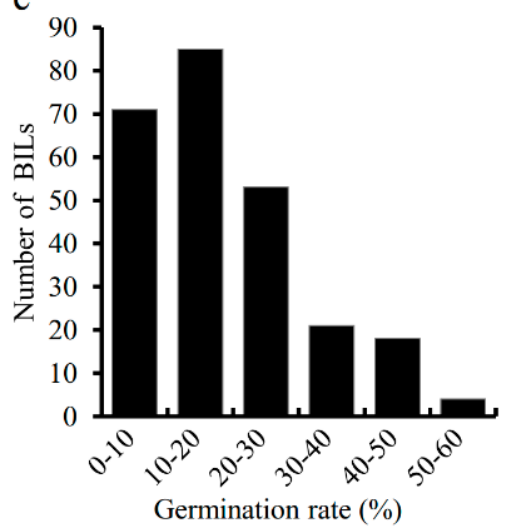

Figure 1. Germination rates of Dongxiang wild rice (DXWR), F6 and their backcross inbred lines (BILs) after aging treatment. (a) the germination rate of DXWR was significantly higher than that of F6 after aging treatment. (b) average germination rates of DXWR and F6 after aging treatment. Significant differences are indicated by asterisks (Student's $t$-test; $p$-value < 0.01). (c) distribution of germination rates in BILs population from crosses DXWR and F6 after aging treatment.

\subsection{BSA-Seq, Data Analysis, and Quality Assessment}

Using whole genome sequencing for the libraries of DXWR, F6, TB-bulk, and IB-bulk, we generated $33,922,707,33,982,607,39,408,702$, and 33,710,561 raw reads, respectively (Table 1). After filtering, we obtained a total of $42.19 \mathrm{~Gb}$ of clean data, including $10.09 \mathrm{~Gb}$ for IB-bulk and $11.79 \mathrm{~Gb}$ for TB-bulk. The total amounts of clean data for the donor parent DXWR and the receptor parent F6 were $10.16 \mathrm{~Gb}$ and $10.14 \mathrm{~Gb}$, respectively. In total, approximately $96.32 \%$ of the clean reads could be aligned to the reference genome. In this study, the average GC content of the four samples was $44.56 \%$, thereby indicating that the GC distribution was normal. Meanwhile, the average depth of the genome coverage of the four samples was $21.50 \times$, while the average genomic coverage was $94.36 \%$ at $1 \times$ coverage and $89.28 \%$ at $5 \times$ coverage. In addition, the $Q$-value $(Q 30)$ was used as the cut-off for quality control. The $Q 30$ values of all samples were $>90 \%$, ranging from $91.54 \%$ (TB-bulk) to $94.70 \%$ (F6), with an average of $93.56 \%$ (Table 1). These results indicated that the data were of good quality and could be used for subsequent analysis.

\subsection{Identification of Candidate Genome Loci for Seed Storability}

After filtering, a total of 330,715 high-quality SNPs and 74,076 high-quality InDels were identified among the different samples. In general, the greater the $\triangle \mathrm{SNP}$-index and $\Delta$ InDel-index, the more likely the contribution of SNPs and InDels to the trait of interest or their association with the candidate genes that control the trait. In this study, the 
$\triangle \mathrm{SNP}$-index and $\Delta \mathrm{InDel}-$ index graphs were plotted and computed against the genome positions (Figure 2). The SNP analysis indicated that the regions containing four genomic loci on chromosome 4 and three genomic loci on chromosome 9 could be the candidate loci associated with seed storability. The seven loci covered a total length of $3.65 \mathrm{Mb}$ and contained a total of 548 genes (Table S2). On the other hand, the InDel analysis indicated that the regions containing five genomic loci on chromosome 4 and one genomic locus on chromosome 9 could contribute to the trait of seed storability. These six loci covered a total length of $3.49 \mathrm{Mb}$ and contained a total of 516 genes (Table S2). Several candidate loci at the intersection of the SNP and InDel analysis were identified at the 18,550,000-20,870,000 bp on chromosome 4 (named as locus-4) and 7,860,000-9,780,000 on chromosome 9 (named as locus-9) and are considered as candidate loci for seed storability of DXWR (Table 2). Within the identified regions, a total of 448 annotated genes were identified (Table 2). Among them, 274 genes had nonsynonymous mutations and 82 genes had frameshift mutations (Table S3).

Table 1. The quality of sequencing data and analysis of sequencing depth and coverage.

\begin{tabular}{|c|c|c|c|c|c|c|c|c|}
\hline & $\begin{array}{l}\text { Raw } \\
\text { Reads }\end{array}$ & $\begin{array}{c}\text { Clean Base } \\
\text { (bp) }\end{array}$ & $\begin{array}{l}\text { Mapping } \\
\text { Rate (\%) }\end{array}$ & Q30 (\%) & GC (\%) & $\begin{array}{c}\text { Average } \\
\text { Depth }(\times)\end{array}$ & $\begin{array}{l}\text { Coverage at } \\
\text { Least } 1 \times(\%)\end{array}$ & $\begin{array}{r}\text { Coverage at } \\
\text { Least } 5 \times(\%)\end{array}$ \\
\hline $\begin{array}{l}\text { Dongxiang wild } \\
\text { rice (DXWR) }\end{array}$ & $33,922,707$ & $10,157,432,212$ & 97.54 & 93.95 & 44.63 & 20 & 97.36 & 93.27 \\
\hline $\begin{array}{c}\text { F6 } \\
\text { Bulked-intolerant }\end{array}$ & $33,982,607$ & $10,144,910,192$ & 96.60 & 94.70 & 45.32 & 21 & 91.06 & 86.93 \\
\hline $\begin{array}{l}\text { BIL samples } \\
\text { (IB-bulk) }\end{array}$ & $33,710,561$ & $10,092,480,184$ & 95.82 & 94.03 & 44.58 & 21 & 94.59 & 88.27 \\
\hline $\begin{array}{l}\text { Bulked-tolerant } \\
\text { BIL samples } \\
\text { (TB-bulk) }\end{array}$ & $39,408,702$ & $11,791,891,500$ & 95.31 & 91.54 & 43.71 & 24 & 94.44 & 88.63 \\
\hline
\end{tabular}
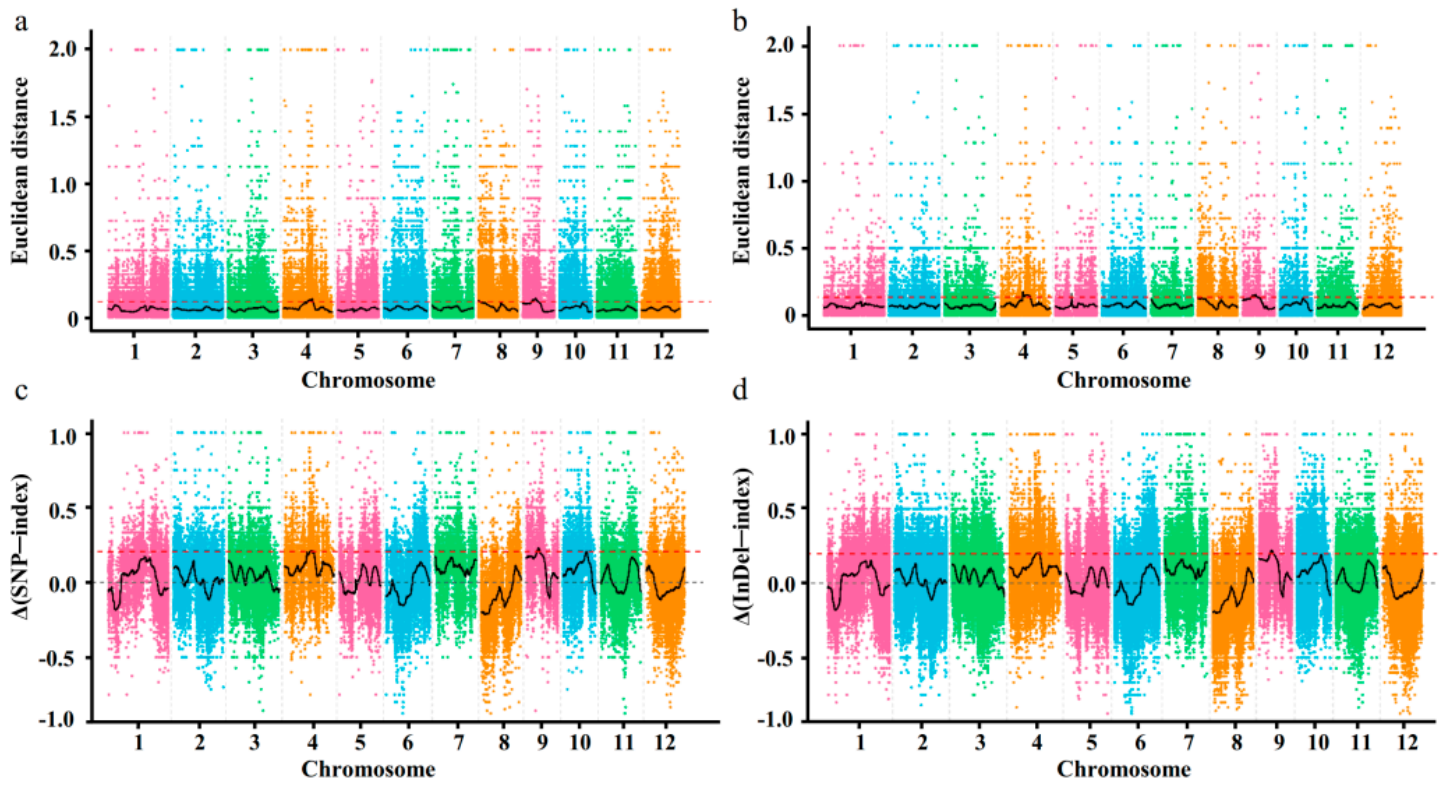

Figure 2. The identifcation of candidate genome regions for seed storability by Euclidean Distance (ED) and deltaindex methods. (a) SNP-ED association algorithm. (b) InDel—ED association algorithm. (c) $\Delta$ (SNP—index) method. (d) $\Delta$ (InDel-index) method. The abscissa is the chromosome number, the colored dot represents the calculated ED value or $\Delta(\mathrm{SNP}$-index $) / \Delta(\mathrm{InDel}$-index $)$ value of the SNP/InDel site, and the black line is the fitted ED value or $\Delta(\mathrm{SNP}-$ index $) / \Delta($ InDel-index $)$ value. The red dashed horizontal lines represent the signifcance association threshold. 
Table 2. Candidate regions of seed storability of DXWR.

\begin{tabular}{ccccc}
\hline Chromosome ID & Start & End & Size (MB) & Gene Number \\
\hline Chr4 & $18,550,000$ & $18,550,000$ & 0.00 & 1 \\
Chr4 & $18,600,000$ & $19,670,000$ & 1.07 & 162 \\
Chr4 & $20,620,000$ & $20,630,000$ & 0.01 & 3 \\
Chr4 & $20,650,000$ & $20,680,000$ & 0.03 & 3 \\
Chr4 & $20,770,000$ & $20,820,000$ & 0.05 & 8 \\
Chr4 & $20,860,000$ & $20,870,000$ & 0.01 & 1 \\
Chr9 & $7,860,000$ & $9,780,000$ & 1.92 & 270 \\
Total & - & - & 3.09 & 448 \\
\hline
\end{tabular}

\subsection{Bioinformatics Analysis of Annotated Genes in the Identified Regions}

To analyse the annotated genes in the identified regions, we carried out bioinformatics analysis using multiple databases. As shown in Table 3, according to the NR database, the candidate regions contained 431 annotated genes, with 265 genes having nonsynonymous mutations and 78 genes having frameshift mutations. However, based on the NT database, we found 448 annotated genes in the candidate loci. Among them, 274 genes had nonsynonymous mutations and 82 genes had frameshift mutations. Meanwhile, we analysed the annotated genes in the Swiss-Prot database and found that 194 genes could be annotated. Among them, 116 genes had nonsynonymous mutations and 28 genes had frameshift mutations. In addition to Swiss-Prot database, we also analysed genes using the TrEMBL database. In total, we found 443 genes in the candidate loci that could be annotated on the TrEMBL database. Among them, 267 genes had nonsynonymous mutations and 78 genes had frameshift mutations. Furthermore, we identified 337 annotated genes in the candidate loci based on the GO database. Among them, 196 genes had nonsynonymous mutations and 53 genes had frameshift mutations.

Table 3. Information of annotated genes in the identified regions using multiple databases.

\begin{tabular}{cccc}
\hline $\begin{array}{c}\text { Annotated } \\
\text { Databases }\end{array}$ & $\begin{array}{c}\text { Gene } \\
\text { Number }\end{array}$ & $\begin{array}{c}\text { Nonsynonymous- } \\
\text { Mutation Gene Number }\end{array}$ & $\begin{array}{c}\text { Frameshift- } \\
\text { Mutation Gene Number }\end{array}$ \\
\hline NR & 431 & 265 & 78 \\
NT & 448 & 274 & 82 \\
TrEMBL & 433 & 267 & 78 \\
SwissProt & 194 & 116 & 28 \\
GO & 337 & 196 & 53 \\
KEGG & 64 & 32 & 4 \\
GOG & 97 & 61 & 15 \\
Total & 448 & 274 & 82 \\
\hline
\end{tabular}

Further, the annotated genes were sorted into GO term categories. The results illustrated that the annotated genes could be clustered into 26, 65, and 74 GO terms in the cellular component (Table S4), molecular function (Table S5), and biological process categories (Table S6), respectively. Within the cellular component category, the cell, organelle, and cell parts were the most abundant. Within the molecular function category, catalytic activity and binding were the most highly represented groups. Metabolic processes and cellular processes were the most abundant in the biological processes category (Figure S1, Table S7). Meanwhile, the annotated genes were also found to be enriched in other stress-related terms, such as biological regulation, stimulus response, nucleic acid binding transcription factor, and transporter activity (Figure S1).

Next, the functional hierarchy of the annotated genes in the KEGG orthology system was classified into five categories, including cellular processes, environmental information processing, genetic information processing, metabolism, and organismal systems. We found annotated 64 genes in the candidate loci based on the KEGG database. Among them, 32 genes had nonsynonymous mutations and four genes had frameshift mutations 
(Table S3). The statistical results showed that most of the annotated genes were associated with metabolism, followed by genetic information processing and cellular processes (Figure S2). Moreover, a KEGG reference pathway analysis showed that the top five enriched pathways were lysine degradation, citrate cycle (TCA cycle), tryptophan metabolism, C5-branched dibasic acid metabolism, and peroxisome (Table S8).

\subsection{Confirmation of the Candidate Loci for Seed Storability}

Using molecular marker background analysis, four BILs (BIL27, BIL49, BIL64, and BIL203) from the DXWR and R974 population were selected to confirm the effect of the candidate loci. In BIL27 and BIL64, segments of chromosome 9 including the candidate locus-9 were substituted with those of DXWR in the background of R974 and no segments including the candidate locus- 4 were substituted (Figure 3). In addition, the segments of chromosome 4 including the candidate locus- 4 were substituted in BIL49 and BIL203 (Figure 3). Meanwhile, BIL49 and BIL203 had no segments including the candidate locus-9 of DXWR (Figure 3). After an artificial aging treatment, the germination rate of R974 was found to be $23.33 \%$, which was significantly lower than that of DXWR. Meanwhile, the germination rates of BIL27, BIL49, BIL64, and BIL203 were $57.50 \%, 54.17 \%, 61.67 \%$, and $50.83 \%$, respectively (Figure 4). Therefore, the germination rates of the four BILs carrying the candidate loci of the seed storability of DXWR were significantly higher than those of R974, implying that the candidate loci could contain the causative genes associated with seed storability in DXWR.
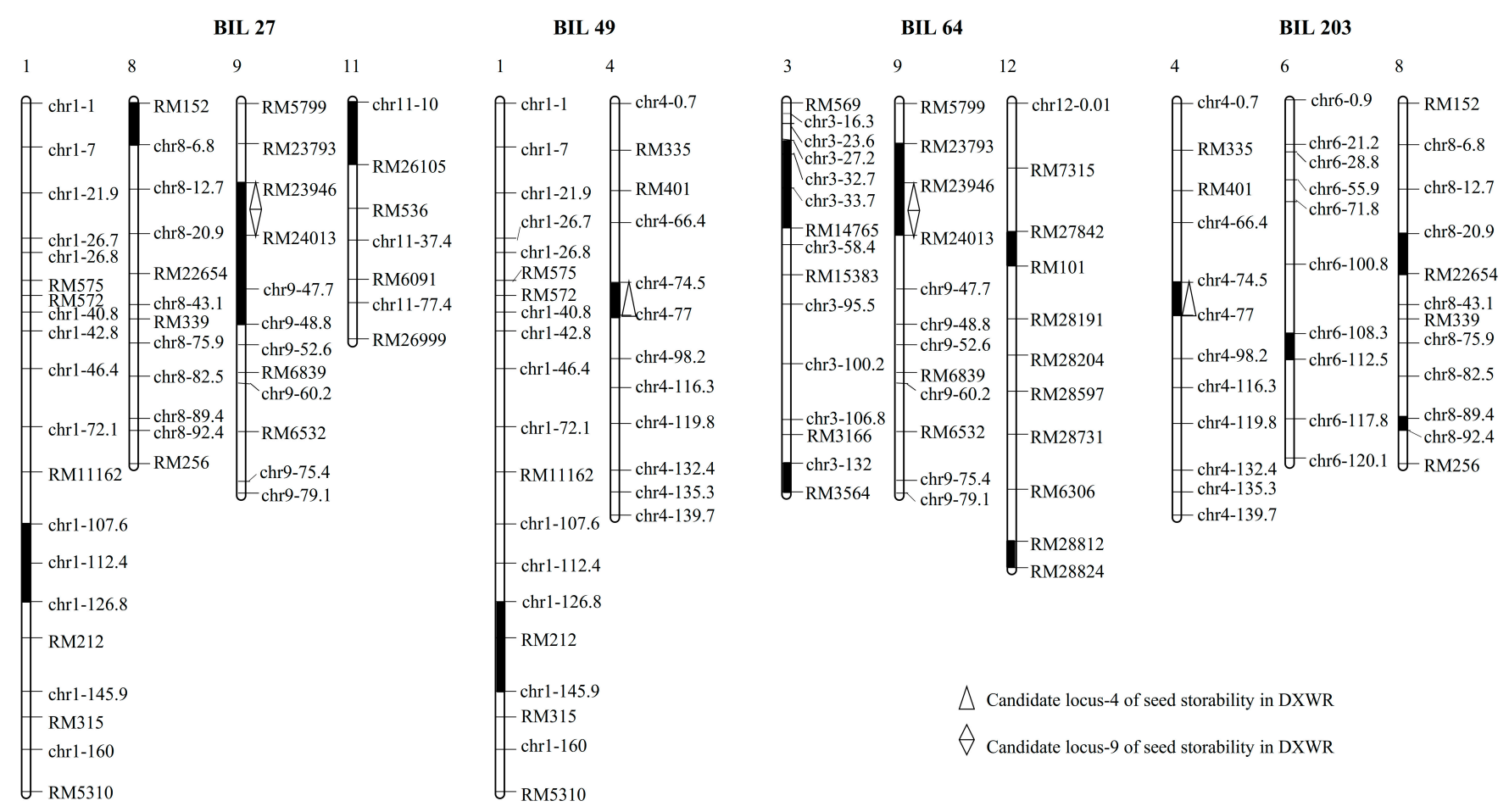

Figure 3. Graphical representation of four BILs (BIL27, BIL49, BIL64, and BIL203) from the DXWR and R974 population. White squares represent homozygous for R974 allele, black squares represent homozygous for DXWR allele. 

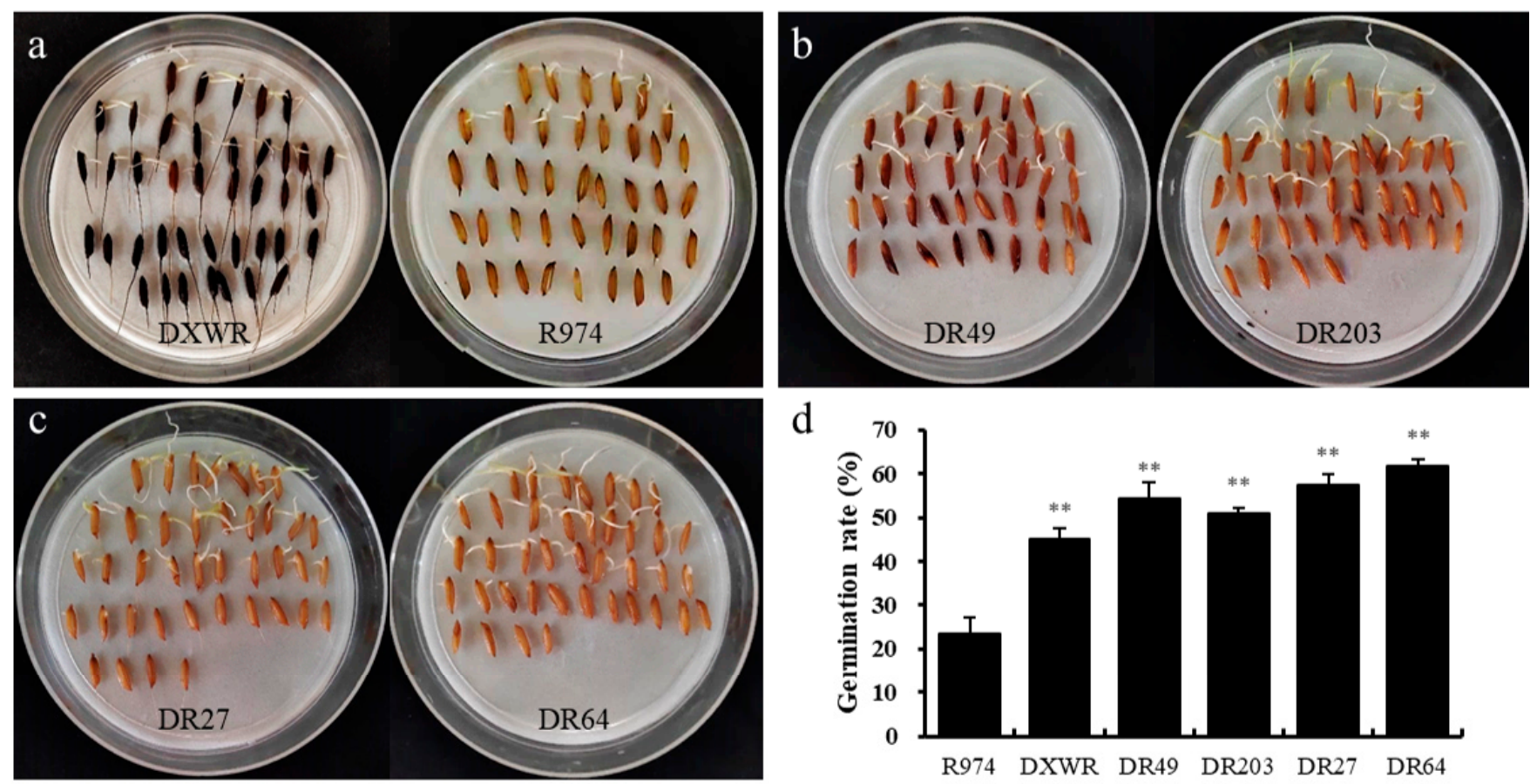

Figure 4. Germination rates of the parents and the four BILs after aging treatment. (a) Comparison of germination rates between DXWR and R974 after aging treatment. (b) phenotype chart of DR49 and DR203. (c) phenotype chart of DR27 and DR64. (d) average germination rates of parents and four BILs after aging treatment. Significant differences are indicated by asterisks (Student's $t$-test; ${ }^{* *} p$-value $<0.01$ ).

\section{Discussion}

In agronomy, the seed storability of rice is an important trait owing to its correlation with seed quality and germination [39]. Thus, understanding the mechanisms of seed storability and breeding varieties with high storability is critical for rice production. Seed storability is a complex quantitative trait that is controlled by large numbers of QTLs [40]. Although some progress has been made in traditional QTL mapping, the underlying molecular mechanisms of seed storability in rice remain poorly defined. Identifying more seed storability-related QTLs from different rice accessions is becoming quite valuable. DXWR has strong seed storability and is an ideal rice material to identify seed storabilityrelated QTLs and illustrate the molecular mechanisms [22]. In this study, we identified the candidate genomic regions on chromosomes 4 and 9 for the seed storability of DXWR. Our results have greatly narrowed the interval for identifying the seed storability-related genes of DXWR and have provided valuable information for the fine-scale mapping and cloning of the seed storability-related genes.

The degree of resistance to aging varies among different rice varieties, which is restricted by nongenetic and genetic factors [41]. Till now, a number of studies have identified many QTLs associated with seed storability in different rice accessions; some of these QTLs were also located on chromosomes 4 and 9. For example, using a set of 85 BILs derived from a cross between Sasanishiki and Habataki, Hang et al. identified a total of 13 seed storability-related QTLs on chromosomes 1, 2, 3, 4, 5, 7, 11, and 12. Among these QTLs, qSSh-4 was mapped between two markers (R288 and C891) on chromosome 4; the existence of $q S S h-4$ was further confirmed by Habataki chromosome segment substitution lines in a Sasanishiki genetic background [42]. Using two different segregating populations, Lin et al. identified seven QTLs for seed storability on chromosomes 1, 2, 5, 6, and 9 . The QTL $q S S n-9$ was simultaneously detected at almost the same region in the interval between RM444 and RM105 on chromosome 9 in the two populations, implying that qSSn-9 was a stable QTL for seed storability [39]. Meanwhile, Li et al. identified 10 QTLs for seed germination percentage using a double haploid population derived from the 
cross between $\mathrm{CJ} 06$ and TN1 during either natural storage or artificial aging. Among the identified QTLs, $q G P-9$ on chromosome 9 was delimited to an interval of $92.8 \mathrm{~kb}$ between two STS markers (P6 and P8) [43]. Furthermore, in some studies, the seed storability-related QTLs were also identified on chromosomes 4 and 9 simultaneously. For example, using 98 BILs derived from a cross between Nipponbare and Kasalath, Miura et al. detected three QTLs for seed longevity, including $q L G-2, q L G-4$, and $q L G-9$ on chromosomes 2, 4 , and 9, respectively, after applying an aging treatment. qLG-4 was located between markers C1100 and C1016 on chromosome 4, and qLG-9 was located between markers C103 and C1751 on chromosome 9 [44]. Subsequently, Sasaki et al. fine mapped qLG-9 into a 30-kb interval of the Nipponbare genome sequence [45]. Using a BIL population derived from a cross between Koshihikari and Kasalath, Li et al. detected six QTLs for seed storability on chromosomes $2,3,4,6,9$, and 11. Among them, qSS-4 was located on chromosome 4 between markers C513 and C1100; qSS-9 was located on chromosome 9 between markers R10783S and S752 [46]. By performing a QTL comparative analysis, the candidate locus-4 and locus-9 identified in our study appeared to partially overlap with $q S S h-4, q S S-9$, and $q S S n-9$. These results imply that these loci could be specific and reliable seed storability-related loci.

A total of 448 annotated genes were identified within our identified loci, of which 274 genes had nonsynonymous mutations and 82 genes had frameshift mutations. The bioinformatics analysis of these annotated genes revealed extensive metabolic activities and cellular processes during seed storability. An in-depth analysis of the biological process annotation of GO revealed that LOC_Os09g13610 was involved in the biological process of jasmonic acid (JA) mediated signalling pathway (GO:0009867). Previous studies had revealed that the hormone JA is a critical regulator of tolerance to abiotic stresses [47,48]. In addition, we found that two genes (LOC_Os04g32020 and LOC_Os09g14670) were involved in the tricarboxylic acid cycle process (GO:0006099), one gene (LOC_Os04g32070) was involved in the oxidation-reduction process (GO:0055114), and one gene (LOC_Os09g15670) was involved in the abiotic stress responses (GO:0009409, GO:0009414). Interestingly, out of the five annotated genes identified in the aforementioned GO database, only LOC_Os09g13610 was also annotated by the KEGG database. Furthermore, the sequence alignment results showed that the locus of LOC_Os09g13610 had many nonsynonymous or frameshift mutations, which differentiated the parents as well as the two bulked pools. These annotated genes could be involved in the regulation of seed storability and need further investigation.

Using genetic background analysis, we selected four BILs from the DXWR and R974 population to analyse the effect of locus- 4 and locus-9. The results showed that the germination rates of the BILs harbouring the identified loci of seed storability were significantly higher than those of R974 after implementing the artificial aging treatments. Therefore, we concluded that the candidate loci identified in our study could contain the genes related to the seed storability of DXWR; additionally, the tightly linked markers of the loci will be valuable in future breeding programs to develop new cultivars with improved rice seed storability.

\section{Conclusions}

In this study, we applied the BSA-seq method and identified the seed storabilityrelated loci of DXWR. By performing a comparative analysis of QTLs, we found that the candidate loci overlapped partially with the seed storability-related QTLs found in previous studies. Meanwhile, 448 annotated genes were identified within the candidate loci, and large numbers of nonsynonymous and frameshift mutations existed in these genes. Additionally, the candidate loci were confirmed by four BILs from the DXWR and R974 population. These results will provide genetic information on the cloning and molecular marker-assisted selection of the seed storability-related genes of DXWR, and thus will be helpful in advancing the breeding strategies and breeding resources required to increase the seed storability of rice. 
Supplementary Materials: The following are available online at https://www.mdpi.com/article/10 .3390 /genes12111831/s1, Figure S1: GO enrichment analysis of annotated genes in the candidate regions. The ordinate is each GO category, the abscissa is the number of genes. Figure S2: KEGG enrichment analysis of annotated genes in the candidate regions. The abscissa is the number of genes annotated to the pathway, and the ordinate is the name of KEGG metabolic pathway. Table S1: The molecular markers used in graphic genotyping of the BILs population of DXWR and R974. Table S2: SNP- and InDel-index association results. Table S3: Nonsynonymous and frameshift mutations in the annotated genes of the identified regions. Table S4: GO analysis in the cellular component category of annotated genes in the identified regions. Table S5: GO analysis in the molecular function category of annotated genes in the identified regions. Table S6: GO analysis in the biological process category of annotated genes in the identified regions. Table S7: GO enrichment analysis of annotated genes in the identified regions. Table S8: KEGG enrichment analysis of annotated genes in the candidate regions.

Author Contributions: M.Z., B.H. and G.D. performed the experiments. M.Z. and W.Y. analyzed the data. Y.C. (Yong Chen) and Y.C. (Yanhong Chen) helped in the sample collection. Y.F. helped to edit the manuscript. M.Z., B.H., Y.F. and F.Z. drafted the manuscript. F.Z. and J.X. contributed to the experimental design and edition of the manuscript. All authors have read and agreed to the published version of the manuscript.

Funding: This research was partially supported by the National Natural Science Foundation of China $(31960370,32070374,31960085,32160467)$, the Natural Science Foundation of Jiangxi Province, China (20202ACB205002), the Foundation of Jiangxi Provincial Key Lab of Protection and Utilization of Subtropical Plant Resources (YRD201903), and the Postgraduate Innovation Fund of Jiangxi Education Department (YC2020-S183).

Institutional Review Board Statement: Not applicable.

Informed Consent Statement: Not applicable.

Data Availability Statement: Not applicable.

Conflicts of Interest: The authors declare no conflict of interest.

\section{References}

1. Yan, W.G.; Li, Y.; Agrama, H.A.; Luo, D.G.; Gao, F.Y.; Lu, X.J.; Ren, G.J. Association mapping of stigma and spikelet characteristics in rice (Oryza sativa L.). Mol. Breed. 2009, 24, 277-292. [CrossRef] [PubMed]

2. Zhang, K.L.; Zhang, Y.; Sun, J.; Meng, J.S.; Tao, J. Deterioration of orthodox seeds during ageing: Influencing factors, physiological alterations and the role of reactive oxygen species. Plant Physiol. Biochem. 2020, 158, 475-485. [CrossRef] [PubMed]

3. Yan, S.; Huang, W.; Gao, J.; Fu, H.; Liu, J. Comparative metabolomic analysis of seed metabolites associated with seed storability in rice (Oryza sativa L.) during natural aging. Plant Physiol. Biochem. 2018, 127, 590-598. [CrossRef]

4. Zhang, H.; Duan, Z.; Li, Y.; Zhao, G.; Zhu, S.; Fu, W.; Peng, T.; Zhao, Q.; Svanberg, S.; Hu, J. Vis/NIR reflectance spectroscopy for hybrid rice variety identification and chlorophyll content evaluation for different nitrogen fertilizer levels. Roy. Soc. Open Sci. 2019, 6, 191132. [CrossRef]

5. Zheng, H.; Zhou, L.; Wei, J.; Tang, Q.; Zou, Y.; Tang, J.; Xu, H. Cover crops and chicken grazing in a winter fallow field improve soil carbon and nitrogen contents and decrease methane emissions. Sci. Rep. 2020, 10, 12607. [CrossRef]

6. Zhang, P.; Wu, H.M.; Zhang, F.; Cao, P.H.; Cai, M.Y.; Song, W.H.; Liu, S.J.; Jiang, L. Improving the storage tolerance of rice seeds by down-regulating OsLOX by RNAi. J. Nanjing Agric. Univ. 2019, 42, 996-1005, (Chinese with English abstract).

7. Zhang, X.; Hina, A.; Song, S.; Kong, J.; Bhat, J.A.; Zhao, T. Whole-genome mapping identified novel "QTL hotspots regions" for seed storability in soybean (Glycine max L.). BMC Genom. 2019, 20, 499. [CrossRef]

8. Harmeet, K.; Petla, B.P.; Kamble, N.U.; Ajeet, S.; Rao, V.; Prafull, S.; Shraboni, G.; Manoj, M. Differentially expressed seed aging responsive heat shock protein OsHSP18.2 implicates in seed vigor; longevity and improves germination and seedling establishment under abiotic stress. Front. Plant Sci. 2015, 6, 713.

9. He, Y.; Cheng, J.; He, Y.; Yang, B.; Cheng, Y.; Yang, C.; Zhang, H.; Wang, Z. Influence of isopropylmalate synthase OsIPMS1 on seed vigour associated with amino acid and energy metabolism in rice. Plant Biotechnol. J. 2019, 17, 322-337. [CrossRef]

10. Lei, M.; Zhu, F.; Li, Z.; Zhang, J.; Li, X.; Dong, J.; Wang, T. Talen-based mutagenesis of lipoxygenase Lox3 enhances the storage tolerance of rice (Oryza sativa) seeds. PLoS ONE 2015, 10, e0143877.

11. Wu, F.; Luo, X.; Wang, L.; Wei, Y.; Li, J.; Xie, H.; Zhang, J.; Xie, G. Genome-wide association study reveals the QTLs for seed storability in world rice core collections. Plants 2021, 10, 812. [CrossRef]

12. Zhang, X.; Zhang, K.; Wu, J.; Guo, N.; Liang, J.; Wang, X.; Cheng, F. QTL-Seq and sequence assembly rapidly mapped the gene BrMYBL2.1 for the purple trait in Brassica rapa. Sci. Rep. 2020, 10, 2328. [CrossRef] [PubMed] 
13. Ficiciyan, A.; Loos, J.; Sievers-Glotzbach, S.; Tscharntke, T. More than yield: Ecosystem services of traditional versus modern crop varieties revisited. Sustainability 2018, 10, 2834. [CrossRef]

14. Liang, T.; Chi, W.; Huang, L.; Qu, M.; Zhang, S.; Chen, Z.Q.; Chen, Z.J.; Tian, D.; Gui, Y.; Chen, X.; et al. Bulked segregant analysis coupled with whole-genome sequencing (BSA-Seq) Mapping identifies a Novel pi21 haplotype conferring basal resistance to rice blast disease. Int. J. Mol. Sci. 2020, 21, 2162. [CrossRef]

15. Kitony, J.K.; Sunohara, H.; Tasaki, M.; Mori, J.I.; Shimazu, A.; Reyes, V.P.; Yasui, H.; Yamagata, Y.; Yoshimura, A.; Yamasaki, M.; et al. Development of an Aus-derived nested association mapping (Aus-NAM) population in rice. Plants 2021, $10,1255$. [CrossRef] [PubMed]

16. Reyes, V.P.; Angeles-Shim, R.B.; Mendioro, M.S.; Manuel, M.C.C.; Lapis, R.S.; Shim, J.; Sunohara, H.; Nishiuchi, S.; Kikuta, M.; Makihara, D.; et al. Marker-assisted introgression and stacking of major QTLs controlling grain number (Gn1a) and number of primary branching (WFP) to NERICA cultivars. Plants 2021, 10, 844. [CrossRef]

17. Wen, J.; Jiang, F.; Weng, Y.; Sun, M.; Shi, X.; Zhou, Y.; Yu, L.; Wu, Z. Identification of heat-tolerance QTLs and high-temperature stress-responsive genes through conventional QTL mapping; QTL-seq and RNA-seq in tomato. BMC Plant Biol. 2019, 19, 398. [CrossRef]

18. Guo, Z.; Cai, L.; Chen, Z.; Wang, R.; Zhang, L.; Guan, S.; Zhang, S.; Ma, W.; Liu, C.; Pan, G. Identification of candidate genes controlling chilling tolerance of rice in the cold region at the booting stage by BSA-Seq and RNA-Seq. R. Soc. Open Sci. 2020, 7, 201081. [CrossRef]

19. Lu, H.; Lin, T.; Klein, J.; Wang, S.; Qi, J.; Zhou, Q. QTL-seq identifies an early flowering QTL located near Flowering Locus T in cucumber. Theor. Appl. Genet. 2014, 127, 1491. [CrossRef] [PubMed]

20. Cui, Y.; Ge, Q.; Zhao, P.; Chen, W.; Sang, X.; Zhao, Y.; Chen, Q.; Wang, H. Rapid mining of candidate genes for verticillium wilt resistance in cotton based on BSA-Seq analysis. Front. Plant Sci. 2021, 12, 703011. [CrossRef]

21. Zhang, F.; Zhou, Y.; Zhang, M.; Luo, X.; Xie, J. Effects of drought stress on global gene expression profile in leaf and root samples of Dongxiang wild rice (Oryza rufipogon). Biosci. Rep. 2017, 37, BSR20160509. [CrossRef] [PubMed]

22. Jiang, J.Y.; Wu, Y.J.; Zhang, C.H.; Jiang, J.P. Preliminary study on storage characteristics of rice seed resources in different evolution stages. Hybrid Rice 2010, 25, 70-72, (Chinese with English abstract).

23. Ren, R.J.; Wang, P.; Wang, L.N.; Su, J.P.; Sun, L.J.; Sun, Y.; Chen, D.F.; Chen, X.W. Os4BGlu14, a monolignol $\beta$-Glucosidase, negatively affects seed longevity by influencing primary metabolism in rice. Plant Mol. Biol. 2020, 104, 513-527. [CrossRef]

24. Takagi, H.; Tamiru, M.; Abe, A.; Yoshida, K.; Uemura, A.; Yaegashi, H. MutMap accelerates breeding of a salt-tolerant rice cultivar. Nat. Biotechnol. 2015, 33, 445-449. [CrossRef]

25. Li, H.; Durbin, R. Fast and accurate short read alignment with Burrows Wheeler Transform. Bioinformatics 2009, 25, 160-164. [CrossRef]

26. Michelmore, R.W.; Paran, I.; Kesseli, R. Identification of markers linked to disease-resistance genes by bulked segregant analysis: A rapid method to detect markers in specific genomic regions by using segregating populations. Proc. Natl. Acad. Sci. USA 1991, 88, 9828-9832. [CrossRef] [PubMed]

27. McKenna, A.; Hanna, M.; Banks, E.; Sivachenko, A.; Cibulskis, K.; Kernytsky, A. The genome analysis toolkit: A mapReduce framework for analyzing next generation DNA sequencing data. Genome Res. 2010, 20, 1297-1303. [CrossRef]

28. Cingolani, P.; Platts, A.; Wangle, L.L.; Coon, M.; Nguyen, T.; Wang, L.; Land, S.J.; Lu, X.; Ruden, D.M. A program for annotating and predicting the effects of single nucleotide polymorphisms, SnpEff: SNPs in the genome of Drosophila melanogaster strain w1118; iso-2; iso-3. Fly 2012, 6, 80-92. [CrossRef]

29. Reumers, J.; De Rijk, P.; Zhao, H.; Liekens, A.; Smeets, D.; Cleary, J.; Van Loo, P.; Van Den Bossche, M.; Catthoor, K.; Sabbe, B.; et al. Optimized filtering reduces the error rate in detecting genomic variants by short-read sequencing. Nat. Biotechnol. 2012, 30, 61-68. [CrossRef]

30. Hill, J.T.; Demarest, B.L.; Bisgrove, B.W.; Gorsi, B.; Yost, H.J. MMAPPR: Mutation mapping analysis pipeline for pooled RNA-seq. Genome Res. 2013, 23, 687-697. [CrossRef]

31. Dokmanic, I.; Parhizkar, R.; Ranieri, J.; Vetterli, M. Euclidean distance matrices: A short walk through theory, algorithms and applications. IEEE Signal. Proc. Mag. 2015, 32, 12-30. [CrossRef]

32. Altschul, S.F.; Madden, T.L.; Schäffer, A.A.; Zhang, J.; Zhanag, Z.; Webb, M. Gapped BLAST and PSI-BLAST: A new generation of protein database search programs. Nucleic Acids Res. 1997, 25, 3389-3402. [CrossRef] [PubMed]

33. Deng, Y.Y.; Li, J.Q.; Wu, S.F.; Zhu, Y.P.; He, F.C. Integrated nr database in protein annotation system and its localization. Comput. Eng. 2006, 32, 71-74.

34. Yip, Y.L.; Famiglietti, M.; Gos, A.; Duek, P.D.; David, F.P.A.; Gateau, A.; Bairoch, A. Annotating single amino acid polymorphisms in the UniProt/Swiss-Prot knowledgebase. Hum. Mutat. 2008, 29, 361-366. [CrossRef]

35. Ashburner, M.; Ball, C.A.; Blake, J.A.; Botstein, D.; Butler, H.; Cherry, J.M. Gene ontology: Tool for the unification of biology. Nat. Genet. 2000, 25, 25-29. [CrossRef]

36. Minoru, K.; Susumu, G.; Shuichi, K.; Yasushi, O.; Masahiro, H. The KEGG resource for deciphering the genome. Nucleic Acids Res. 2004, 32, 277-280.

37. Tatusov, R.L.; Galperin, M.Y.; Natale, D.A.; Koonin, E.V. The COG database: A tool for genome scale analysis of protein functions and evolution. Nucleic Acids Res. 2000, 28, 33-36. [CrossRef] [PubMed] 
38. Zhu, P.; Wang, C.; Huang, K.; Luo, Y.; Xu, W. A novel pretreatment-free duplex chamber digital PCR detection system for the absolute quantitation of gmo samples. Int. J. Mol. Sci. 2016, 17, 402. [CrossRef]

39. Lin, Q.Y.; Wang, W.Y.; Ren, Y.K.; Jiang, Y.; Sun, A.; Qian, Y.; Zhang, Y.; He, N.; Hang, N.T.; Liu, Z.; et al. Genetic dissection of seed storability using two different populations with a same parent rice cultivar N22. Breed. Sci. 2015, 65, 411-419. [CrossRef]

40. Yuan, Z.; Fan, K.; Xia, L.; Ding, X.; Tian, L.; Sun, W.; He, H.; Yu, S. Genetic dissection of seed storability and validation of candidate gene associated with antioxidant capability in rice (Oryza sativa L.). Int. J. Mol. Sci. 2019, 20, 4442. [CrossRef]

41. Quilloy, F.A.; Labaco, B.; Casal, C.; Dixit, S. Crop establishment in direct-seeded rice: Traits, physiology, and genetics. In Rice Improvement; Ali, J., Wani, S.H., Eds.; Springer: Cham, Switzerland, 2021; pp. 171-202.

42. Hang, N.T.; Lin, Q.Y.; Liu, L.L.; Liu, X. Mapping QTLs related to rice seed storability under natural and artificial aging storage conditions. Euphytica 2015, 203, 673-681. [CrossRef]

43. Li, C.S.; Shao, G.S.; Wang, L.; Wang, Z.F.; Zhang, H.S. QTL identification and fine mapping for seed storability in rice (Oryza sativa L.). Euphytica 2017, 213, 127. [CrossRef]

44. Miura, K.; Lin, S.; Yano, M.; Nagamine, T. Mapping quantitative trait loci controlling seed longevity in rice (Oryza sativa L.). Theor Appl. Genet. 2002, 104, 981-986. [CrossRef]

45. Sasaki, K.; Takeuchi, Y.; Miura, K.; Yamaguchi, T.; Ando, T.; Ebitani, T.; Higashitani, A.; Yamaya, T.; Yano, M.; Sato, T. Fine mapping of a major quantitative trait locus, $q L G-9$, that controls seed longevity in rice (Oryza sativa L.). Theor. Appl. Genet. 2015, 128, 769-778. [CrossRef]

46. Li, L.; Lin, Q.; Liu, S.; Liu, X.; Wang, W.; Hang, N.T.; Liu, F.; Zhao, Z.; Jiang, L.; Wan, J. Identification of quantitative trait loci for seed storability in rice (Oryza sativa L.). Plant. Breed. 2012, 131, 739-743. [CrossRef]

47. Wang, Y.; Mostafa, S.; Zeng, W.; Jin, B. Function and mechanism of jasmonic acid in plant responses to abiotic and biotic stresses. Int. J. Mol. Sci. 2021, 22, 8568. [CrossRef]

48. Singh, P.; Mukhopadhyay, K. Comprehensive molecular dissection of TIFY Transcription factors reveal their dynamic responses to biotic and abiotic stress in whea (Triticum aestivum L.). Sci. Rep. 2021, 11, 9739. [CrossRef] [PubMed] 